\title{
The Abstracts of the Papers J. IIlum. Engng. Inst. Jpn. Vol. 95, 2011
}

\section{No. 2 February}

\section{CONTENTS}

\section{Original Paper:}

Development of Non-invasive Blood Glucose Monitoring System based on Infrared Spectroscopy

81

S. KOYAMA and H. ISHIZAWA

\section{Letter:}

The Estimation of Images of Foods under Spot Lighting Technique for Store Illumination-Influences of Light Source Color-

87

H. FUJITA, Y. NAKASHIMA, M. TAKAMATSU and $M$. OOTA

\section{ABSTRACTS}

Development of Non-invasive Blood Glucose Monitoring System based on Infrared Spectroscopy

Shouhei KOYAMA and Hiroaki ISHIZAWA

Over the past few decades, an increasing number of studies have been done on the measurement of blood glucose. We developed a noninvasive system to measure blood glucose based on infrared spectroscopy (IR). With this system, we were able to measure the spectrum of the human finger by using Fourier transform infrared spectroscopy-attenuated total reflectance (FTIR-ATR). Furthermore, by using an individual calibration model based on partial least squares regression, we were able to measure human blood glucose. We describe the measurement results and assess the validity of the new system in this paper.

KEYWORDS: non-invasive measurement, self-monitoring blood glucose, personal calibration model fourier transform infrared spectroscopy, partial least squares regression

The Estimation of Images of Foods under Spot Lighting Technique for Store Illumination-Influences of Light Source Color-

Hiroki FUJITA, Yoshio NAKASHIMA, Mamoru TAKAMATSU and Masaaki OOTA

KEYWORDS: store illumination, suitable lighting system, factor analysis, image evaluation

\section{No. 5 May}

Original Papers:

Clinical Application of Non-invasive Blood Glucose Monitoring system by Infrared Spectroscopy

251

The Influence of the Cathode Heating System of the Inverter Ballast on the Position of the Cathode Spot 255 T. UETSUKI, K.MAEHARA, Y. GENBA and T. KANDA

Qualitative Analysis of Dyed Textile Cultural Property by Laser-Induced Fluorescence 260

S. TOKUTAKE, T. DOZONO, and H. ISHIZAWA

\section{ABSTRACTS}

Clinical Application of Non-invasive Blood Glucose Monitoring System by Infrared Spectroscopy

Shouhei KOYAMA, Yuki MIYAUCHI and Hiroaki ISHIZAWA

Recently, diabetics have been steadily increase, they have to measure the blood glucose again and again a day. We invented a non-invasive measurement system of blood glucose based on an Infrared Spectroscopy of attenuated total reflection. And, using PLS calibration model could measure the blood glucose of diabetics. The purpose is to examine the accuracy of this monitoring system in clinical trial. And the difference of reflection times of infrared in ATR prism was examined.

KEYWORDS: non-invasive measurement, blood glucose of diabetic, error grid analysis, fourier transform infrared spectroscopy, clinical application

The Influence of the Cathode Heating System of the Inverter Ballast on the Position of the Cathode Spot Tadao UETSUKI, Kenji MAEHARA, Yuki GENBA and Takashi KANDA

Recently the fluorescent lamps having a higher output and longer life, have been developed in the field of residential lighting. If lamp life can be estimated by measuring electric signals such as lamp current and cathode heating current, the estimation would be very useful for designing lamp operating systems. To estimate lamp life, it is very important to know where the 
light-spot position, which is the spot that emits the electrons, exists on the cathode. It was reported that the light-spot position for one operating system could be located by measuring the electronic signals. However there are many kinds of the electronic circuits for use in fluorescent lamp operating system. It has not been confirmed whether this method for locating the lightspot position is useful for the other kind of electronic circuits. In this paper, authors reveal that the method mentioned above is useful and that the way of heating the cathode in the electronic circuit influences how the spot moves as burning time passes.

KEYWORDS: cathode spot, cathode heating, FL, inverter ballast

Qualitative Analysis of Dyed Textile Cultural Property by Laser-Induced Fluorescence

Shota TOKUTAKE, Tomomi DOZONO, and Hiroaki ISHIZAWA

A qualitative analysis of dyed textile cultural property by spectroscopic measurement is described. An analysis by non-destructive measurement is desired in the interest of conserving of cultural property. However, the existing methods are based on destructive inspection, and a lot of time and proficiency is necessary for analysis. To improve those problems, the application of laser-induced fluorescence (LIF) as a non-destructive measurement technique is proposed in this study. We measured the LIF spectrum of a sample, and compared it with a data base. This measurement technique is nondestructive, and easy.

KEYWORDS: dyed textile cultural property, non-destructive measurement, laser-induced fluorescence

\section{No. 8 August}

\section{CONTENTS}

\section{Original Papers}

Quantum Efficiency Measurement for Lamp Phosphors in accordance with Radiometric Standards 431 K. OHKUBO and Y. NAKAGAWA

The Effect of Season on Impression of Lighting Part1 within -and between-subject variation 439 K. ISHIDA, Y. INOUE, H. UTIYAMA and J. KURATA

\section{Letters}

An Interior Illuminance Calculation Method for LED Light Sources

A. UCHIDA, F. HIRENZAKI and Y. OHTANI
Identification and Quantitative Analysis of Fiber Mixtures by Infrared Spectroscopy 450 T. DOZONO, H. ISHIZAWA, S. TOKUTAKE and T. MIKI

\section{ABSTRACTS}

Quantum Efficiency Measurement for Lamp Phosphors in accordance with Radiometric Standards Kazuaki OHKUBO and Yasuo NAKAGAWA

To measure the quantum efficiencies of lamp phosphors, a JCSS (Japan Calibration Service System) traceable measurement method that uses a polychromator improved optical design to reduce stray light, and an integrating sphere has been developed. To verify the measurement accuracy of this method, a monochromator, which has a $0-45^{\circ}$ incident optical system, was used. The BRDF (Bidirectional Reflectance Distribution Function) of the phosphor was measured by a goniophotometer. A comparison was made between these JCSS traceable measuring devices on the $260-400 \mathrm{~nm}$ wavelength characteristics of the internal quantum efficiency of the blue phosphor BAM $\left(\mathrm{BaMgAl}_{10} \mathrm{O}_{17} \mathrm{EU}^{2+}\right)$, which has excitation characteristics up to $400 \mathrm{~nm}$. The results agreed within $4 \%$ of the difference of the measured values. This is a good agreement considering that it was a composite measure and that the uncertainty of the spectral irradiance standard in the spectral region is lower than 3\%. It has also been established that the proposed method using a polychromator and an integrating sphere shows sufficient measurement accuracy.

KEYWORDS: fluorescent, quantum efficiency, lamp phosphor, ;plychromator, integrating sphere

The Effect of Season on Impression of Lighting: Part1 Within -and between-subject variation Kyoko ISHIDA, Youko INOUE, Hironobu UTIYAMA and Junichi KURATA

This study investigates how the season and the correlated color temperature affected the impression of lighting. However, subjective evaluation inevitably has individual variation. Therefore the purpose of this paper is to clarify how within -and between- subject variation changes. The experiment variables are the season, the illuminance of room and the correlated color temperature of fluorescent lamp. The season are summer and winter, there are 12 levels of vertical illuminance of the room from 0.5 to $2000 \mathrm{~lx}$, and the correlated color temperature of lamps are 3000 and $6700 \mathrm{~K}$. The subjects are 27 to 31 young women. They evaluate brightness, glare, comfort and relaxation effect of the lighting. In conclusion; 1) Brightness, glare and comfortable sensa- 
tions change depending on the illuminance. The influence on the relaxation effect due to the illuminance is small, and the relation is clear in each individual. The subjects have suitable illuminance levels for relaxation. 2) All of four items are influenced by the change of illuminance level in between-subject variation. The range of illuminance in which the evaluation between subject is stabilized is as follows; brightness is $500 \mathrm{~lx}$ or more, glare is $50 \mathrm{~lx}$ or less, comfort is from 20 to $50 \mathrm{~lx}$, and relaxation is from 10 to $20 \mathrm{~lx}$. 3) The variation between individuals is larger than the variation individual, though there is a little difference with changes in illuminance.

KEYWORDS: season, color temperature, illuminance, individual variation, brightness, comfort

An Interior Illuminance Calculation Method for LED Light Sources

Akira UCHIDA, Fumihiko HIRENZAKI and Yoshihiko OHTANI

KEYWORDS: LED (Light Emitting Diode), illuminance calculation, Monte Carlo method

Identification and Quantitative Analysis of Fiber Mixtures by Infrared Spectroscopy

Tomomi DOZONO, Hiroaki ISHIZAWA, Shota TOKUTAKE and Takashi MIKI

KEYWORDS: composition of fibers, nondestructive analysis, infrared spectroscopy, SIMCA, PLSR

\section{No. 11 November}

\section{CONTENTS}

\section{Original Papers:}

Study about Increasing Brightness using Task-ambient Lighting in Offices 699

T. KOTANI, Y. NAKAMURA, S. KANAYA and Y. MIKI

Appropriate Washstand Lighting for Applying Makeup

711

S. OKUDA, S. YAMAGUCHI, N. HARA and W. IWAI

Effect of Excitation Frequency on Breakdown Voltage in Narrow Cold Cathode Tube under Ne Discharge 718 M. GOTO and T. ARAI

Dependence of Electric Characteristics of Magneticcoupling-type Electrodeless Discharge Lamp on Excitation Coil Turns 722

\section{ABSTRACTS}

Study about Increasing Brightness using Task-ambient Lighting in Offices

Tomoko KOTANI, Yoshiki NAKAMURA, Sueko KANAYA and Yasuhiro Miki

We conducted subjective experiments to determine an ideal method for achieving both energy saving and comfort in office lighting. The experiments were conducted in an actual office under 48 conditions of lighting and furniture, such as different average illuminance, lighted areas, reflectance, and height of partitions, and added wall-washers. Luminance images were captured from the subject's viewpoint, and converted into brightness images based on the Nakamura method. We then analyzed the relationship between the perceived brightness and the brightness images. In the results, the subjective evaluation value of the brightness of the room strongly correlated with the average values of the brightness scale of vertical components, such as a wall or partition, calculated from the brightness images. This suggests that we can reduce the electricity consumption of lighting and achieve higher perceived brightness without losing comfort by raising the brightness of the vertical components in the field of view.

KEYWORDS: office lighting, task and ambient lighting, energy saving, brightness, visual comfort, luminance distribution

Appropriate Washstand Lighting for Applying Makeup Shino OKUDA, Sayaka YAMAGUCHI, Naoya HARA and Wataru IWAI

This study aims to determine the appropriate lighting conditions for applying makeup. We presumed that applying makeup includes two tasks: 'base makeup', i.e., foundation and cheek rouge; and 'point makeup', i.e., mascara and lip rouge. The brightness or visibility of one's face, and the task performance of applying makeup in different lighting conditions is reported. We conducted a subjective experiment on the lighting condition of a washstand in a bathroom. The washstand used in the experiment was equipped with four fluorescent lamps or two incandescent lamps, enabling varying conditions of illuminance on the face, light color, and lighting of the mirror to be presented. Twenty subjects observed their faces under 32 lighting conditions, and they evaluated the "brightness of one's face", "visibility of features of one's face", "visibility of shadows", "glare of lighting", "discomfort of glare", "annoyance of shadow", "task performance", and "satisfaction" after applying both base makeup and point makeup. In the evalu- 
ation results, "task performance" and "satisfaction" in base makeup were clearly higher when the illuminance on the face was high. "Task performance" and "satisfaction" in point makeup were also clearly higher when the mirror was lit from all four sides or from vertical sides by fluorescent lamps than when the lighting was from above and below.

KEYWORDS: makeup, task performance, annoyance of shadow, washstand, lighting position

\section{Effect of Excitation Frequency on Breakdown Voltage in Narrow Cold Cathode Tube under Ne Discharge Miki GOTO and Toshihiko ARAI}

We investigated the discharge characteristics in a cold cathode lamp of $4 \mathrm{~mm}$ inner diameter used for backlighting liquid crystal displays. The electrode on one side of the lamp was made of nickel, and that on other side was platinum. The Ne gas pressure filling the lamp was $53 \mathrm{hPa}$. The cathode voltages of a lamp with the electrodes made of different materials were simultaneously measured with a high voltage probe connected to a digital oscilloscope when the lamp was operated by a sine wave power supply under the same discharge condition. The breakdown and maintained voltages in the case of the nickel electrode were lower than those of the platinum electrode. The measured reignition voltages were almost constant breakdown voltages in the frequency region of 0.1 to $100 \mathrm{~Hz}$. In frequencies above $100 \mathrm{~Hz}$, the reignition voltages decreased sharply, a becoming close to the maintained voltages. The accurate breakdown voltage was estimated to be less than a $100 \mathrm{~Hz}$ sine wave.

KEYWORDS: cold cathode, LCD backlighting, break- down voltage, Ne discharge, Ne metastable atom, life time

Dependence of Electric Characteristics of Magneticcoupling-type Electrodeless Discharge Lamp on Excitation Coil Turns

Tomohiro YAMAGUCHI, Tomonori SAKUMA and Yoshio WATANABE

The electric characteristics of a magnetic-couplingtype electrodeless discharge lamp are investigated. The discharge tube of the lamp is a hollow cylinder, and a magnetic path made of a ferrite core passes through the tube. A high-frequency power voltage is applied across an excitation coil wound around the core through a capacitor. The operating frequency is adjusted to the resonance frequency of the circuit. The light emission from the lamp and magnetic loss are measured as a function of the number of excitation coil turns. The resonance frequency and light output from the lamp decrease with increase of the number of coil turns, while the coil input power shows nearly constant characteristics. The light output decreases with the number of coil turns due to increment of magnetic loss. The eddy current loss in the magnetic material is independent of the operating frequency, but the hysteresis loss that comprise a large part of the magnetic loss around $150 \mathrm{kHz}$ increases with decrease of the operation frequency. These results show that higher frequency is preferable for operating an inductively coupled electrodeless lamp with a magnetic core.

KEYWORDS: electrodeles discharge lamp, ferrite core, magnetic coupling 